\title{
Broken or socially-mistuned mirror neurons in autism? An investigation via transcranial magnetic stimulation
}

Jellina Prinsen ${ }^{1,2^{*}} \&$ Kaat Alaerts ${ }^{1,2}$

${ }^{1}$ Neuromodulation Lab - Neurorehabilitation Research Group, Department of Rehabilitation Sciences, KU Leuven, Leuven, Belgium.

2 Leuven Autism Research Consortium (LAuRes), KU Leuven, Belgium

* Correspondence:

Jellina Prinsen

Tervuursevest 101, box 1501

3001 Leuven, Belgium

jellina.prinsen@kuleuven.be

Number of words: 5100

Number of tables: 2

Number of figures: 1

References: 54 


\section{Abstract}

Individuals with an autism spectrum disorder (ASD) experience persistent difficulties during social interactions and communication. Previously, it has been suggested that deficits in the so-called 'mirror neuron system' (MNS), active during both action execution and observation, may underlie these social difficulties. It is still a topic of debate however whether deficiencies in the simulation of others' actions (i.e. "broken" mirroring) forms a general feature of ASD, or whether these mostly reflect a lack of social attunement. The latter would suggest an overall intact MNS, but an impaired modulation of MNS activity according to variable social contexts. In this study, 25 adults with ASD and 28 age- and IQ-matched control participants underwent transcranial magnetic stimulation (TMS) during the observation of hand movements under variable conditions. Hand movements were presented via a live interaction partner, either without social context to assess basic motor mirroring, or in combination with direct and averted gaze from the acting model to assess socially modulated mirroring. Overall, no significant group differences were revealed, indicating no differential in MNS activity in ASD, compared to controls. Interestingly however, regression analyses revealed that, among ASD participants, higher social symptom severity was associated with both reduced basic motor mirroring and aberrant socially modulated mirroring (i.e. no enhancement of MNS activity upon direct vs. averted gaze). These findings further challenge the notion that MNS dysfunctions constitute a principal feature of ASD, but that variations in MNS function are related to differential expressions of (social) symptom severity.

Keywords: autism spectrum disorder (ASD), transcranial magnetic stimulation (TMS), mirror neuron system (MNS), eye contact. 


\section{Introduction}

Autism spectrum disorders (ASD) are a set of neurodevelopmental disorders characterized by severe impairments in reciprocal social interaction and communication, combined with a reduced and restricted pattern of interests and behaviors (DSM-5; American Psychiatric Association, 2013). Although it is now generally accepted that ASD characteristics emerge as a result of abnormal neural development, much debate persists regarding the exact nature of its neural etiology. One prominent explanatory model, known as the Broken Mirror Theory, suggests that abnormalities within the so-called 'mirror neuron system' (MNS) may underlie the socio-communicative difficulties in ASD (lacoboni \& Dapretto, 2006; Oberman \& Ramachandran, 2007). The human MNS can be defined as a set of brain regions that are activated not only when participants perform a particular action, but also when they simply observe movements performed by other persons (Cattaneo \& Rizzolatti, 2009). By directly simulating observed actions onto the observer's own motor system, the MNS is considered to play a key role in the automatic and effortless understanding of others' actions, as well as associated socio-cognitive processes such as imitation, intention understanding and empathy (lacoboni, 2009; Rizzolatti \& Sinigaglia, 2010), several of which are impaired in ASD (Boria et al., 2009; Kana, Wadsworth, \& Travers, 2011). Accordingly, it has been suggested that dysfunctions within the MNS might contribute to poor social understanding in ASD.

Neural MNS activity during action observation can be assessed using a range of neuroscientific methods, including functional and structural neuroimaging, magneto- or electroencephalographic (M/EEG) suppression indices of mu waves in the 8-13 $\mathrm{Hz}$ band over the sensorimotor strip, and transcranial magnetic stimulation (TMS) indicators of corticospinal excitability. Together, these methods have shown less brain activity (Dapretto et al., 2006; Grèzes, Wicker, Berthoz, \& de Gelder, 2009; Martineau, Andersson, Barthélémy, Cottier, \& Destrieux, 2010) and reduced cortical thickness (Hadjikhani, Joseph, Snyder, \& Tager-Flusberg, 2006) in brain areas belonging to the MNS, and diminished mu suppression (Bernier, Dawson, Webb, \& Murias, 2007; Dumas, Soussignan, Hugueville, Martinerie, \& Nadel, 2014; Nishitani, Avikainen, \& Hari, 2004; Oberman et al., 2005) and corticospinal excitability (Enticott et al., 2012; Théoret et al., 2005) during the observation of others' movements in participants with ASD. Other studies however reported no such deficits (fMRI: Marsh \& Hamilton, 2011; Pokorny et al., 2015; EEG: Fan, Decety, Yang, Liu, \& Cheng, 2010; Raymaekers, Wiersema, \& Roeyers, 2009; Ruysschaert, Warreyn, Wiersema, Oostra, \& Roeyers, 2014; TMS: Enticott et al., 2013), indicating that evidence for a general Broken Mirrors account is mixed (see Hamilton, 2013 for a review). 
Of relevance, aberrant mirror responses in ASD have mainly been reported when observing social or emotionally-loaded stimuli (e.g. Dapretto et al., 2006; Grèzes et al., 2009), whereas normal MNS responses are often observed when viewing simple or goal-directed actions (e.g. Marsh and Hamilton, 2011). This mixed pattern of MNS activations in ASD can be interpreted in light of the social-top down response modulation (STORM) model of imitation as proposed by Wang and Hamilton (2012) (taking into consideration that imitative skills are grounded within the MNS; lacoboni, 2009), which posits that social cues determine whether a particular action should be mimicked or not. This notion was experimentally confirmed in a series of behavioral studies focusing on gaze cues, showing that mimicry of hand actions is faster when eye-to-eye contact is established between actor and observer socially relevant context), compared to situations in which the actor displayed an averted eye gaze (socially irrelevant context) (Wang, Newport, \& Hamilton, 2011). When applied to ASD, STORM proposes that imitation itself may be intact in persons with ASD, but that they - in specific social situations - lack appropriate modulatory responses according to the social demands of that situation (Hamilton, 2013). Especially in the case of eye-to-eye contact, hypothesized to be experienced as unpleasant and/or not socially salient or rewarding in ASD (Senju \& Johnson, 2009; Tanaka \& Sung, 2016), these gaze cues could interfere with an appropriate top-down control. Indeed, a further investigation of the social modulation of mimicry by gaze cues showed overall intact imitation abilities, but aberrant imitation responses in reaction to direct gaze cues in participants with ASD (Forbes, Wang, \& Hamilton, 2017).

Recently, a series of TMS studies extended this behavioral work by showing that activity within the MNS is enhanced when observed movements are accompanied by the actor's direct compared to averted gaze (Prinsen \& Alaerts, 2019, 2020; Prinsen et al., 2017; Prinsen, Brams, \& Alaerts, 2018), thereby providing support to the notion that the MNS underlies the encountered gaze-related modulations of mimicry in the Wang experiments. Single-pulse TMS is a non-invasive brain stimulation method commonly adopted in the context of action observation research, during which the primary motor cortex (M1) is magnetically stimulated to assess its excitability under various observational conditions. In general, the observation of others' actions results in a significant enhancement of M1 excitability, which is considered a result of excitatory connections between M1 and the MNS (Fadiga, Craighero, \& Olivier, 2005). The demonstration of higher M1 facilitation upon movements combined with direct eye contact provided an important neurophysiological basis for the proposed top-down modulation of the MNS as postulated by Wang and Hamilton (2012). However, direct neurophysiological evidence in favor of an inappropriate modulation within ASD upon eye-to-eye contact, i.e. paralleling the behavioral results by Forbes et al. (2017), is at the moment still lacking. 
In sum, previous studies evaluating MNS activity in ASD have reported mixed results concerning its (dys)function, which appear to be dependent on the social or emotional content of the stimulus. Indirectly, this seems to be in line with an intact but socially mistuned MNS as proposed by the STORM model, instead of a generally broken MNS as advanced by the Broken Mirror Theory. A principal aim of the current study was to investigate the extent of putative MNS deficits in adults with ASD and assess the relevance of both accounts, as well as to extend prior TMS work in neurotypical individuals (Prinsen \& Alaerts, 2019, 2020; Prinsen et al., 2017) and behavioral work in individuals with ASD (Forbes et al., 2017) to obtain a neurophysiological investigation of socially modulated mirroring in ASD. To do so, we used TMS for probing potential group differences in MNS activity during observation of simple hand movements. To address the general motoric aspects of the MNS in ASD (basic motor mirroring), hand movements were presented against a white background without any further social context. To investigate the role of the social context on MNS activity in ASD (socially modulated mirroring), hand movements were combined with the interaction partner gazing either directly towards the participant, or gazing away from the participant. In order to enhance the ecological validity of our experiment, gaze and movement cues were conveyed by a live interaction partner (Prinsen \& Alaerts, 2019). In line with the STORM account, we expected basic motor mirroring to be unaffected in ASD, but predicted deficits in socially modulated mirroring depending on observed gaze cues (i.e. no enhancement of M1 excitability upon perceiving movements combined with interaction partner's direct compared to averted gaze), as demonstrated previously at the behavioral level (Forbes et al., 2017). 


\section{Method and Materials}

\subsection{Participants}

Participants were 25 young adult men, aged between 18-35 years, with a clinically established diagnosis of ASD according to DSM-IV or DSM-5 criteria (American Psychiatric Association, 2013) and 28 age- and IQ-matched non-ASD participants (see table 1 for participant demographics). All participants were right-handed (confirmed with the Edinburgh Handedness Questionnaire; Oldfield, 1971), met safety criteria for TMS (Rossi, Hallett, Rossini, \& Pascual-Leone, 2012) and provided written informed consent. Additional exclusion criteria for non-ASD participants included the presence of neurological illness (e.g. stroke, epilepsy, concussion) or psychiatric conditions (e.g. ASD, ADHD). Ethical approval was granted by the local ethics committee at the University of Leuven in accordance to the Declaration of Helsinki (World Medical Association, 2013). Reported comorbid disorders within ASD participants included ADHD/ADD (4 participants), depression (3), burnout (1), dyslexia (1), dyscalculia (1), anxiety problems (1) and Gilles de la Tourette (1). Six of the ASD participants reported stable use of psychoactive medication (antipsychotics, antidepressants and/or psychostimulants) for at least three months at the time of study enrollment.

Self-report assessments of ASD symptomatology confirmed the presence of significantly higher autism-related impairments in ASD participants, as demonstrated on all subtests of the Autism Questionnaire (AQ; Baron-Cohen, Wheelwright, Skinner, Martin, \& Clubley, 2001) and the Social Responsiveness Scale (SRS; Constantino and Todd, 2005) (table 1). There were no significant group differences in terms of age or in verbal and/or performance abilities as assessed by four subtests of the Dutch Wechsler Adult Intelligence Scale, fourth edition (WAIS-IV-NL; Wechsler, 2012; table 1). Note that individuals were recruited to participate in a larger project investigating the effects of oxytocin treatment for ASD (not part of the current report). 


\section{Table 1. Participant demographics.}

\begin{tabular}{|c|c|c|c|}
\hline & $\begin{array}{l}\text { ASD group } \\
(\mathrm{n}=25) \\
\text { Mean (SD) }\end{array}$ & $\begin{array}{l}\text { Comparison group } \\
(\mathrm{n}=28) \\
\text { Mean (SD) }\end{array}$ & $\begin{array}{l}\text { Independent } \\
\text { samples } t \text { test }\end{array}$ \\
\hline Age in years: months & $22: 4(4: 1)$ & $21: 6(2: 10)$ & 0.84 \\
\hline AQ Total Score (raw) & $26.84(7.23)$ & $12.43(5.49)$ & 8.22 *** \\
\hline Social skills & $4.48(2.65)$ & $1.25(1.45)$ & $5.58^{* * *}$ \\
\hline Attention switching & $6.24(1.92)$ & $3.25(1.48)$ & $6.38^{* * *}$ \\
\hline Attention to detail & $5.92(2.27)$ & $3.32(2.34)$ & $4.09 * * *$ \\
\hline Communication & $5.12(2.42)$ & $1.68(1.31)$ & $6.54 * * *$ \\
\hline Imagination & $5.08(2.17)$ & $2.93(1.90)$ & $3.84^{* * *}$ \\
\hline SRS Total Score (raw) & $71.76(29.29)$ & 35.28 (13.29) & $5.94^{* * *}$ \\
\hline Social awareness & $20.00(9.29)$ & $10.64(4.38)$ & $4.77^{* * *}$ \\
\hline Social motivation & $23.64(11.92)$ & $10.50(6.00)$ & $5.15^{* * *}$ \\
\hline Social communication & $13.20(5.64)$ & $6.64(2.82)$ & $5.44^{* * *}$ \\
\hline Repetitive behavior & $14.92(5.88)$ & $7.50(3.85)$ & $5.48^{* * *}$ \\
\hline \multicolumn{4}{|c|}{ WAIS-IV-NL subtest (scaled) } \\
\hline Block Patterns & $13.20(3.77)$ & $13.36(2.31)$ & -0.18 \\
\hline Similarities & $10.76(3.44)$ & $11.46(2.32)$ & -0.88 \\
\hline Matrices & $11.04(2.95)$ & $11.32(2.39)$ & -0.38 \\
\hline Vocabulary & $10.60(3.42)$ & $11.43(2.63)$ & -0.99 \\
\hline \multicolumn{4}{|l|}{ MNS parameters at rest } \\
\hline rMT (in \%MO) & $43.28(7.51)$ & $42.18(7.47)$ & 0.53 \\
\hline Baseline CSE (in mV) & $1.82(1.90)$ & $2.10(1.55)$ & -0.59 \\
\hline
\end{tabular}

AQ: Autism Quotient; SRS: Social Responsiveness Scale; WAIS-IV-NL: Wechsler Adult Intelligence Scale, fourth edition (Dutch version); MNS: mirror neuron system; rMT: resting motor threshold; \%MO: percentage of maximum stimulator output; CSE: corticospinal excitability; ${ }^{* * *} p<.001$

\subsection{TMS and EMG set-up and apparatus}

MNS activity was assessed by administering single-pulse TMS to the left primary motor cortex (M1) using a Magstim-200 stimulator (Magstim Company Ltd., UK) with a hand-held $70 \mathrm{~mm}$ figure-of-eight coil positioned against the scalp. Optimal coil location for magnetic stimulation ('hotspot') was identified as the scalp position that produced maximal motor evoked potentials (MEPs) in the contralateral first dorsal interosseous (FDI) while at rest, recorded via electromyography (EMG). The resting motor threshold (rMT), expressed as a percentage of the maximum stimulator output (\%MO), was individually defined as the lowest 
stimulation intensity that produced MEPs of at least $50 \mathrm{mV}$ in 5 out of 10 consecutive trials. Resulting rMT values did not differ between groups (table 1). The FDI muscle of the index finger was selected as it is intrinsically implicated in the to-be-observed index finger movements; and the MNS simulates observed actions in a strictly muscle-congruent fashion (Fadiga, Fogassi, Pavesi, \& Rizzolatti, 1995). During assessment, MEPs were also collected from the abductor minimi digiti (ADM) control muscle, which is not implicated in the observed movements. Due to the overlap of hand muscle representations in $M 1$, stimulation parameters are assumed to be satisfactory for assessing condition-specific modulations simultaneously for both muscles (Gentner \& Classen, 2006; Krings, Naujokat, \& Graf v. Keyserlingk, 1998). MEP amplitudes were recorded via surface EMG using disposable Ag$\mathrm{AgCl}$ adhesive electrodes (Kendall Medi-Trace) arranged in a tendon-belly montage with the reference electrode attached to the wrist. Signal software (version 6.02, Cambridge Electronic Design, UK) in combination with a CED Micro 1401 mk II analog-to-digital converting unit (Cambridge Electronic Design, UK) was used for triggering of the TMSstimulator and the recording of the EMG signal (amplified, sampled at $2000 \mathrm{~Hz}$ and $5-1000$ $\mathrm{Hz}$ band-pass filtered).

\subsection{Experimental procedure and stimuli}

Participants were seated approximately $80 \mathrm{~cm}$ from an opaque voltage-sensitive liquid crystal (LC) shutter screen $(20 \times 30 \mathrm{~cm}$; DreamGlass Group, Spain) attached to a black panel. Following determination of the individual's hotspot and rMT, participants were administered ten TMS pulses at 130\% rMT while at rest (with 4 second interval) to provide a baseline measure of FDI corticospinal excitability (CSE). As recommended by Oberman et al. (2016), formal testing indicated no difference between the ASD and comparison group (table 1), indicating no low-level differences in M1 excitability.

Next, movement observation trials were presented through the transparent LC screen via a female stimulus person seated behind the panel. Adopted movements were similar to those used in previous studies (Prinsen \& Alaerts, 2019, 2020), involving index finger abduction movements of a horizontally held right hand (dorsal view) in three different contexts. To investigate basic motor mirroring, hand movements were presented against a white background, without the stimulus person being visible to the observing participant. To address socially modulated mirroring, movements were presented in combination with the stimulus person gazing directly towards the observing participants vs. with the stimulus person averting her face and gazing $30^{\circ}$ to the right. Previous studies in neurotypical participants have consistently demonstrated enhanced MNS activity upon observation of actions combined with direct vs. averted eye gaze from the acting model (Prinsen \& Alaerts, 
2019, 2020; Prinsen et al., 2017). Each trial was of 4 seconds duration. All conditions were presented 5 times in blocks of 4 trials (i.e. 20 MEPs per condition) with an inter-trial interval of 2 seconds in a pseudo-random sequence. In between trials and blocks, the LC screen resumed the opaque state.

TMS pulses during the experimental procedure were delivered at $130 \%$ of the subject's rMT. Coinciding with the TMS pulse on the third second of stimulus presentation, the stimulus person performed the index finger abduction movement (similar set-up as in Prinsen \& Alaerts, 2019, 2020). In order to ensure that all participants viewed and attended the stimuli properly, they were asked once at a random time point during the TMS assessment to verbally report the type of stimulus that was presented in the previous trial.

\subsection{Data handling and analysis}

Peak-to-peak amplitudes of the TMS-induced MEPs were determined using in-house MATLAB scripts (version R2015a, MathWorks Inc., USA). Trials in evidence of excessive background EMG activity (i.e. conditional mean \pm 2.5 SD) in the 110-10 millisecond time interval prior to TMS administration, indicative of voluntary movements and/or heightened muscle tone, were removed. Trials were also removed if MEP amplitudes exceeded 1.5 interquartile distances from the conditional mean (in total: $10.87 \%$ of all trials).

Since no group difference in baseline CSE was encountered (table 1), and consistent with previous TMS studies (Enticott et al., 2013, 2012), MNS activity during movement observation trials was putatively measured using a MEP percentage change variable $(\mathrm{MEP} \%)$ relative to the average MEP response while at rest: $\left[\left(\mathrm{MEP}_{\text {condition }}-\mathrm{MEP}_{\text {rest }}\right) /\right.$ $\left.\left.M P_{\text {rest }}\right) \times 100\right]$. A higher MEP\% score is indicative of more MNS activity. Since Shapiro Wilk's tests $(W)$ indicated non-normal distributions, MEP\% values were logarithmic transformed. As a log-transformation cannot be performed for negative values (and some MEP\% values were negative), prior to the transformation we added a constant of 100 to each of the values to ensure that they were all positive (similar procedure as Enticott et al., 2013, 2012). After transformation, normality was obtained for all variables ( $W p>.07)$. Note that for illustrative purposes, figures in the Results section display the untransformed MEP\% values.

For all log-transformed MEP\% scores, single-sample $t$-tests were performed, separately for each participant group and investigated hand muscle, to assess whether MEPs recorded during movement observation were significantly higher compared to rest. Next, group differences in basic motor mirroring were assessed by subjecting the corresponding logtransformed MEP\% scores to an analysis of variance (ANOVA) including the betweensubject factor Group (ASD vs. non-ASD) and the within-subject factor Muscle (FDI and 
ADM). Group differences in socially modulated mirroring were assessed by subjecting the corresponding MEP\% data to an ANOVA including the factors Group and Muscle, as well as the within-subject factor Model's Gaze (averted and direct). Planned comparisons were conducted to specifically compare MEPs recorded upon direct vs. averted gaze for each muscle per group. Finally, in order to explore the contribution of several predictor variables (age, SRS total score, AQ total score) on the dependent variables of interest, step-wise multiple regression analyses (with forward selection: $F$ to enter $=1, F$ to remove $=0$ ) were conducted.

All statistics were calculated with Statistica 10 (StatSoft, USA). Results were considered significant with a $p$-value lower than .05. Where appropriate, the partial Eta square $\left(\eta_{\mathrm{p}}^{2}\right)$ value or the coefficient of determination $\left(R^{2}\right)$ are given as an estimate of effect size.

\subsection{Secondary outcome measure: gaze behavior}

To ensure that differences in overt visuospatial attention did not underlie any putative MNS differences, eye tracking was performed by means of head-mounted SMI eye-tracking glasses (pupil camera sampling rate: $30 \mathrm{~Hz}$, binocular tracking) and SMI iView acquisition software (version 1.2; SensoMotoric Instruments, Germany). Spatial resolution and gaze position accuracy for the SMI eye tracking device are estimated at $0.1^{\circ}$ and $0.5^{\circ}$ at ideal conditions, respectively. Glasses were adjusted to the participant's comfort and a three-point calibration procedure (as implemented in iView software package) was performed before recording. Due to technical failure or low quality recordings (e.g. due to slippage of the headmounted glasses or excessive head movements), eye tracking data were not available for 11 participants (4 ASD participants and 7 non-ASD participants).

The SMI BeGaze software package (version 3.3) was used for the analysis of the recorded eye movements. From the raw eye tracking data, fixations were detected by means of the built-in SMI dispersion based algorithm with the recommended parameters (i.e. minimum fixation duration: $80 \mathrm{~ms}$; maximum dispersion values: $100 \mathrm{px}$ ). Taking the total dwell time into account (i.e. sum of durations of all detected fixations and saccades during the experimental gaze trials), an overall tracking ratio of $84.80 \%$ for the ASD group and $80.36 \%$ for the comparison group was obtained. Fixations were assigned to area's of interest (AOl's) positioned over the eyes and hand region via the Semantic Gaze Mapping procedure implemented in BeGaze. Fixations that were not assigned to one of these AOls were labelled as "outside AOI". Besides the number of fixations (i.e. fixation count), proportional looking times (\%) for each AOI were analyzed, which were calculated as a function of the total registered dwell time (i.e. compared to the number of trails in which fixations were successfully detected by the built-in algorithm; ASD group range: $15-20$ trials; non-ASD 
group range: 12 - 20 trials). Both outcome measures were separately analyzed using ANOVAs including the between-subject factor Group (ASD vs. non-ASD) and the withinsubject factors AOI (hand and eyes region) and Model's Gaze (averted and direct).

In terms of proportional looking times towards the predefined AOls, the ANOVA indicated no main effect of Group $\left(F(1,42)=0.26, p=.62, \eta_{p}^{2}=.006\right)$, nor two- or three-way interaction effects with this factor (all $p>$.24). Also for the absolute number of fixations to each AOI, no effect of Group $\left(F(1,42)<.001, p=.98, \eta^{2}{ }_{p}<.001\right)$ or any significant interactions with this factor (all $p>$.56) were revealed. This suggests that both ASD and non-ASD participants displayed similar gaze behaviors during the experiment. Furthermore, significant $A O I \times$ Gaze interactions were encountered, both in terms of proportional looking times $(F(1,42)=36.46, p$ $\left.<.001, \eta^{2} p=.47\right)$ as the number of fixations $\left(F(1,42)=26.16, p<.001, \eta^{2}=.38\right)$, indicating that participants with and without ASD mainly favored the Hand AOI in the averted gaze condition, whereas proportional looking times and fixation counts were equally spread across AOls in the direct gaze conditions. These findings imply that, within both groups, it is unlikely that the stimulus person's averted gaze induced gaze following and, in turn, shifted spatial attention away from the to-be-observed hand movements. 


\section{$3 \quad$ Results}

\subsection{Basic motor mirroring}

Single-sample $t$-tests against zero showed that MEP amplitudes of the experimental FDI were significantly enhanced compared to rest (i.e. MEP\% > 0) in both the ASD and comparison group, indicating significant observation-induced MNS activity in both groups during the observation of simple hand movements (table 2). MEPs were also enhanced compared to rest in the control ADM muscle of the ASD group, but not significantly in nonASD participants. Yet, the overall ANOVA indicated no main effects of Group $(F(1,50)=1.39$, $\left.p=.24, \eta^{2}{ }_{p}=.03\right)$ or Muscle $\left(F(1,50)=0.62, p=.43, \eta^{2} p=.01\right)$, nor a Group $\times$ Muscle interaction effect $\left(F(1,50)<.01, p=.94, \eta^{2} p<.001\right)$ (figure 1A).

Table 2. MEP\% amplitudes (indexing \% change from rest).

\begin{tabular}{ccccc}
\hline & \multicolumn{2}{c}{ ASD group $(\boldsymbol{n}=\mathbf{2 5})$} & \multicolumn{2}{c}{ Comparison group $(\boldsymbol{n}=\mathbf{2 8})$} \\
& Mean (SD) & $\boldsymbol{t}$ against 0 & Mean (SD) & $\boldsymbol{t}$ against 0 \\
\hline Basic motor mirroring & & & & \\
FDI MEP\% & $38.37(82.27)$ & $t=2.33^{*}$ & $21.22(52.78)$ & $t=2.09$ * \\
ADM MEP\% & $29.13(60.68)$ & $t=2.40^{*}$ & $19.91(63.45)$ & $t=1.55$ \\
Socially modulated mirroring & & & & \\
FDI MEP\% averted gaze & $38.23(88.76)$ & $t=2.15^{*}$ & $22.07(63.22)$ & $t=1.85$ \\
MEP\% direct gaze & $67.93(138.79)$ & $t=2.45^{*}$ & $30.20(54.22)$ & $t=2.95^{* *}$ \\
ADM MEP\% averted gaze & $25.23(60.70)$ & $t=1.80$ & $18.70(69.29)$ & $t=1.43$ \\
MEP\% direct gaze & $44.04(70.23)$ & $t=3.14^{* *}$ & $24.23(62.25)$ & $t=2.05$ \\
\hline
\end{tabular}

FDI: first dorsal interosseous; ADM: abductor digiti minimi; MEP: motor evoked potential; ${ }^{*} p<.05 ;{ }^{* *} p$ $<.01$

\subsection{Socially modulated mirroring}

During the observation of movements combined with different gaze cues, the ANOVA revealed a significant effect of observed gaze direction $\left(F(1,50)=12.29, p<.001, \eta^{2}{ }_{p}=.20\right)$, indicating that across groups and muscles, perceived direct gaze elicited significantly higher MEPs (relative to rest) compared to averted gaze. There was no significant main effect of Group $\left(F(1,50)=1.34, p=.25, \eta^{2} p=.03\right)$ or Muscle $\left(F(1,50)=1.05, p=.31, \eta^{2} p=.02\right)$, and no interaction effect between Gaze $\times$ Group $\left(F(1,50)=1.19, p=.28, \eta^{2} p=.02\right)$ or between Muscle $\times$ Group $\left(F(1,50)=0.01, p=.92, \eta^{2} p<.001\right)$. In contrast to previous studies from our lab (Prinsen \& Alaerts, 2019, 2020; Prinsen et al., 2017, 2018), there was no Muscle $\times$ Gaze 
interaction $\left(F(1,50)=0.10, p=.75, \eta_{p}^{2}=.002\right)$. Also the three-way interaction between these factors was not significant $\left(F(1,50)=0.81, p=.37, \eta^{2}{ }_{p}=.02\right)$.

Further inspection of the data by means of planned comparisons suggests the possibility of a less specific muscle activation pattern mainly in the ASD group, as they showed significant gaze-related modulations in both the experimental FDI (planned comparisons: $t(24)=-2.58$, $p=.02)$ and control ADM muscle $(t(24)=-2.71, p=.009)$. Non-ASD participants on the other hand only demonstrated a significant gaze effect in the FDI $(t(27)=-2.11, p=.04)$, but not the ADM muscle $(t(27)=-0.95, p=.35)$ (see also the single-sample $t$-test against zero in table 2). However, since the overall ANOVA interaction terms were not significant, caution is advised when interpreting these effects.

\subsection{Relationship between MNS and ASD symptomatology}

Basic motor mirroring. For MEPs collected during observation of simple index finger movements, the step-wise forward regression model indicated a significant effect of SRS total score $\left(F(1,22)=10.02, p=.004, R^{2}=.36\right)$. Inspection of the beta coefficient $(\beta=-.90)$ revealed a negative association between self-reported social impairments and the extent that simple hand movements are simulated by the observer's MNS (figure 1B). The other remaining variables (age, $A Q$ total score) were not allowed to enter the model.

Socially modulated mirroring. In order to quantify gaze-related modulations during action observation, a similar MEP\% score as described previously was calculated to capture the extent to which observed direct gaze elicits higher MEPs relative to the MEP response during averted gaze: $\left[\left(\left(M P_{\text {direct }}-M P_{\text {averted }}\right) / M P_{\text {averted }}\right) \times 100\right]$. The regression model revealed a significant effect of SRS total score herein $\left(F(1,23)=6.33, p=.02, R^{2}=.21\right)$. Examination of the beta coefficient $(\beta=-.46$; figure 1D) suggested that the ability to show socially adaptive gaze-related modulations of MNS activity is associated with less self-reported impairments in the social domain within the ASD group.

Note that no significant associations were identified in the comparison group (none of the predictor variables were allowed to enter the model; i.e. $F$ to enter $<1$ ). 
A. Basic motor mirroring

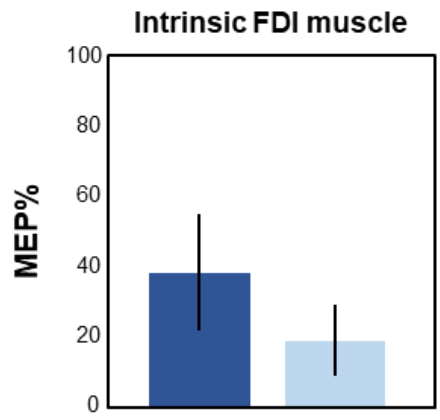

ASC NT
Control ADM muscle

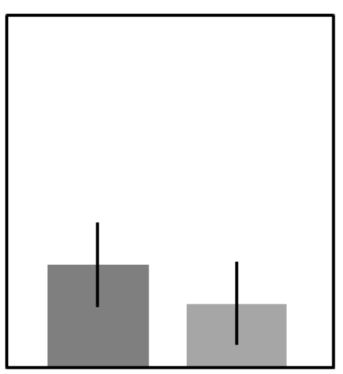

ASC NT

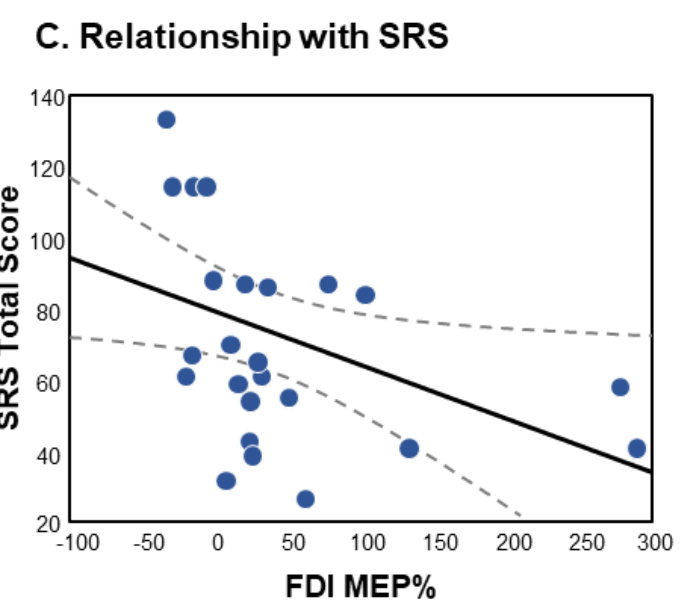

D. Relationship with SRS

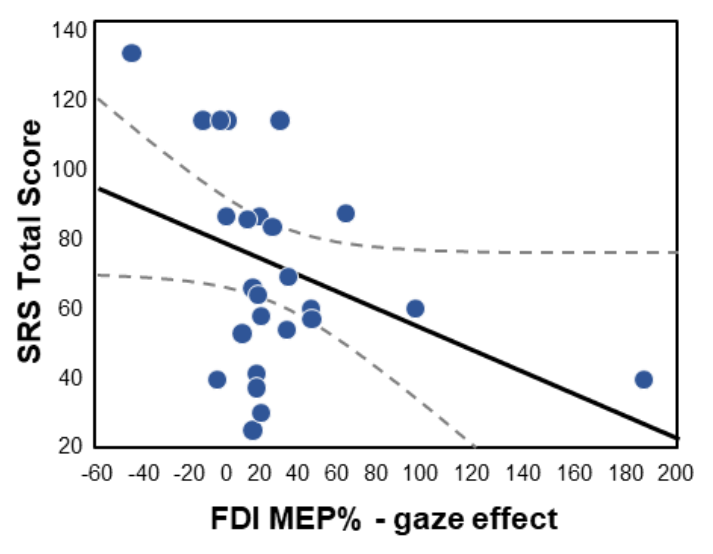

Figure 1. (A) Average ( \pm SE) MEP\% values (indexing \% change from rest) for each group and muscle during simple action observation. A higher value is indicative of enhanced MNS activity. No significant group differences were encountered. (B) Average ( \pm SE) MEP\% values (\% change from rest) for each muscle, group and gaze condition during action observation in combination with direct vs. averted gaze cues from the actor. Contrary to expectations, results indicate significant gaze-related modulations in both participant groups. (C-D) Scatterplots demonstrating the significant negative relationship between FDI MEP\% scores during observation of simple finger movements $(C)$ or FDI MEP\% scores capturing gaze-related modulations (\% change in MEP during direct versus averted gaze) (D) and ASD symptom severity, as measured by the Social Responsiveness Scale (SRS). Dotted lines denote $95 \% \mathrm{Cl}$. 


\section{Discussion}

Individuals with ASD have impairments in social communication and interaction (American Psychiatric Association, 2013). In line with the hypothesized role of the mirror neurons system (MNS) in several important social abilities (e.g. action perception, intention understanding and empathy), the Broken Mirror Theory (Oberman \& Ramachandran, 2007) was put forward, suggesting that a dysfunction of the MNS could result in some of the social symptoms of ASD. However, others proposed that these impairments may be due to difficulties in using social cues to adapt MNS activity to the demands of the social environment (Wang \& Hamilton, 2012). Using transcranial magnetic stimulation (TMS), the current study investigated the relevance of both accounts by examining neurophysiological MNS activity (quantified as the amplitude of TMS-induced MEPS) in individuals with ASD compared to a group of age- and IQ-matched control participants under various observational conditions.

To address the possibility of a global MNS deficit in ASD when simulating others' actions, we investigated basic motor mirroring during the observation of another person's hand movements. In accordance with a body of EEG (Fan et al., 2010; Raymaekers et al., 2009; Ruysschaert et al., 2014), neuroimaging (Marsh \& Hamilton, 2011; Pokorny et al., 2015) and TMS studies (Enticott et al., 2013) reporting no group differences during simple action observation, individuals with ASD showed no evidence of reduced MNS activity. In addition, the analysis of several TMS parameters when at rest revealed no low-level dysfunction of M1 excitability in ASD (assessed via rMT and baseline CSE in table 1), suggesting that M1 and its corticospinal projections are not primarily affected in ASD (see also Oberman et al., 2016; Théoret et al., 2005 for convergent findings). Together, these findings challenge a strong of "broken mirroring" as a principal hallmark of ASD.

Other studies evaluating MNS function in ASD provided indications that putative impairments are dependent on the socio-emotional content of the stimulus (Dapretto et al., 2006; Grèzes et al., 2009). These findings are in accordance with a neurophysiological extension of the behavioral STORM account of imitation by Wang \& Hamilton (2012), positing that individuals with ASD may rely differently on socio-emotional cues to determine when it is adaptive to simulate others' movements. However, adopting several types of tasks and stimuli, not all studies have found group differences (e.g. Bastiaansen et al., 2011; Schulte-Rüther et al., 2017), thereby highlighting the necessity to discover the different social conditions under which MNS impairments are evident in ASD. Here, we combined the observation of simple hand movements with direct and averted gaze cues from the acting model to investigate putative differences in socially modulated mirroring between ASD and control participants. 
Since difficulties with eye-to-eye contact are often evident in ASD (Senju \& Johnson, 2009; Tanaka \& Sung, 2016) and previous behavioral investigations indicated aberrant gazerelated modulations in behavioral imitation responses in ASD (Forbes et al., 2017), we expected deficits in socially modulated mirroring depending on observed gaze cues. Contrary to our expectations however, individuals with ASD showed - at the level of the group - no evidence of reduced MNS activity during the observation of hand movements in combination with eye contact. Also note that similar gaze behaviors were recorded for both participant groups, thereby rendering it unlikely that differences in overt attention underlie the (lack of) differences in MNS activity between groups.

It is however important to note that, among ASD participants, self-reported social impairments as measured by the Social Responsiveness Scale (SRS) were significantly associated with both basic motor mirroring and socially modulated mirroring. Specifically, more social impairments were related with diminished motor mirroring upon perceiving simple hand movements, and less socially adaptive gaze-related modulations in MNS activity (i.e. no enhanced mirroring upon action observation in combination with direct vs. averted gaze cues from the model). These findings are in line with previous studies reporting an association with social symptom severity (TMS: Enticott et al., 2012; EEG: Fan et al., 2010; fMRI: Wadsworth, Maximo, Donnelly, \& Kana, 2018), suggesting that various functional markers of the MNS may be able to reflect the level of social impairments in ASD. This notion is further underscored by recent EEG studies by Hudac et al. $(2015,2017)$, who demonstrated that children and adults with distinct genotypes of ASD were differentially impacted in terms of mu rhythm suppression in response to social vs. non-social stimuli. Rather than affecting all individuals with ASD, MNS impairments may indicate a neurobiological subtype of ASD that is associated with more severe social symptomatology. These findings are of particular importance in the current pursuit of distinct neurobiological subtypes of ASD (e.g. Bertelsen et al., 2021).

Taken together, this and other works demonstrate that there are specific social situations in which MNS function during action observation may be compromised in (some) individuals with ASD. Related to the neurobiological basis for these impairments in (socially modulated) mirroring, the STORM model proposes that the top-down control over the MNS originates in brain regions involved in the evaluation of the social features in the current interaction (Wang \& Hamilton, 2012). This notion was experimentally confirmed in a neural modelling analysis demonstrating a modulatory impact of observed gaze cues on the effective connectivity the medial prefrontal cortex and the superior temporal sulcus (the main visual input region of the MNS) during action observation (Wang, Ramsey, \& Hamilton, 2011). Accordingly, several brain imaging studies have shown aberrant structural (Fishman, Datko, Cabrera, Carper, \& 
Müller, 2015) and functional (Fishman, Keown, Lincoln, Pineda, \& Müller, 2014) connections between the MNS and social brain regions in participants with ASD, as well as an impaired top-down modulation of the EEG-based mu response during action observation (Dumas et al., 2014). In this view, the MNS may be conceived as the end-state region of a neural circuit signaling whether or not the observed actions are to be simulated. Further investigations adopting effective connectivity measures during action observation might shed further light on the source (e.g. "broken" mirror neurons or atypical neural connectivity) and the functional significance of the encountered MNS impairments in ASD. This will allow us to decide whether the MNS should be a possible target for diagnosis, subtyping or treatment in ASD, or may be considered an epiphenomenon of other brain mechanisms.

Finally, subtle differences were noted in the muscle-specificity of recorded MEPs between groups. In general, TMS-induced MEPs are selectively enhanced for those muscles that are naturally involved in the observed movements, as initially demonstrated by Fadiga et al. (1995) and later replicated extensively (for a review, see Fadiga et al., 2005). Results within control participants were largely consistent with this principle, indicating enhanced MEPs (compared to rest) and gaze-related effects in the index finger muscle, but not the little finger muscle, during the observation of index finger movements. For participants with ASD however, the pattern of results was overall less muscle-specific. Although these findings seem to suggest the possibility of an impaired muscle-matching mechanism in individuals with ASD (see also Théoret et al., 2005, for similar findings during basic action observation), the overall interaction terms capturing the muscle factor were not significant (raising the possibility of a Type II error). Further TMS studies specifically designed to address these subtle differences in muscle-matching - i.e. adopting at least two to-be-observed movements, optimized stimulation sites per movement and simultaneous MEP recordings from all involved muscles - are necessary to confirm this notion. Another speculative possibility could entail that, especially within a salient experimental setting involving a live interaction partner, MNS activity in ASD is more arousal-driven. In line with the behavioral findings by Forbes et al. (2017), showing faster reaction times for mimicry of both 'to-mimic' and 'not-to-mimic' movements during direct gaze from the acting model, this might suggest that individuals with ASD may be sensitive to direct gaze as a general alerting signal, but that it is not used to specifically modulate MNS activity in a cost-effective way. 


\section{Conclusion}

In sum, our results indicate no significant group differences, not in terms of basic motor mirroring nor in terms of socially modulated mirroring, thereby providing further indications that MNS dysfunctions do not constitute a principal hallmark of ASD. Notably however, interindividual variability in ASD social symptom severity (as measured by the Social Responsiveness Scale) was significantly related to both a diminished mirror response upon simple action observation and a lack of gaze-related modulations upon perceiving direct vs. averted gaze cues from the interaction partner. These findings are of particular importance in the current pursuit of distinct neurobiological subtypes of ASC.

\section{Acknowledgements}

The authors wish to thank all those who took part in the study. Furthermore, we also wish to thank Lotte Engels, Edith Claus, Valérie Vermeersch and Antje Wynants for their assistance during data collection, prof. Jean Steyeart and the members of the Leuven Autism Research Consortium for their help in participant recruitment, and Paul Meugens and prof. Stephan P. Swinnen for their methodological and technical support.

This research was supported by grants from the Flanders Fund for Scientific Research (FWO [KAN 1506716N, G079017N]) and the Branco Weiss fellowship of the Society in Science ETH Zurich granted to KA. JP is supported by a FWO junior postdoctoral fellowship $(1257621 N)$ and the Marguerite-Marie Delacroix foundation (GV/B-335).

\section{Conflict of interest statement}

The authors of this study declare no conflict of interest. 


\section{References}

American Psychiatric Association. (2013). Diagnostic and statistical manual of mental disorders (5th ed.). Washington DC; USA: American Psychiatric Publishing.

Baron-Cohen, S., Wheelwright, S., Skinner, R., Martin, J., \& Clubley, E. (2001). The AutismSpectrum Quotient (AQ): Evidence from Asperger Syndrome/high-functioning Autism, males and females, scientists and mathematicians. Journal of Autism and Developmental Disorders, 31(1), 5-17. https://doi.org/10.1023/A:1005653411471

Bastiaansen, J. A., Thioux, M., Nanetti, L., Van Der Gaag, C., Ketelaars, C., Minderaa, R., \& Keysers, C. (2011). Age-related increase in inferior frontal gyrus activity and social functioning in autism spectrum disorder. Biological Psychiatry, 69(9), 832-838. https://doi.org/10.1016/j.biopsych.2010.11.007

Bernier, R., Dawson, G., Webb, S., \& Murias, M. (2007). EEG mu rhythm and imitation impairments in individuals with autism spectrum disorder. Brain and Cognition, 64(3), 228-237. https://doi.org/10.1016/j.bandc.2007.03.004

Bertelsen, N., Landi, I., Bethlehem, R. A. I., Seidlitz, J., Busuoli, E. M., Mandelli, V., ... Lombardo, M. V. (2021). Imbalanced social-communicative and restricted repetitive behavior subtypes of autism spectrum disorder exhibit different neural circuitry. Communications Biology, 4(1), 574. https://doi.org/10.1038/s42003-021-02015-2

Boria, S., Fabbri-Destro, M., Cattaneo, L., Sparaci, L., Sinigaglia, C., Santelli, E., ... Rizzolatti, G. (2009). Intention understanding in autism. PLOS ONE, 4(5), e5596. https://doi.org/10.1371/journal.pone.0005596

Cattaneo, L., \& Rizzolatti, G. (2009). The mirror neuron system. Archives of Neurology, 66(5), 557-560. https://doi.org/10.1001/archneurol.2009.41

Constantino, J. N., \& Todd, R. D. (2005). Intergenerational transmission of subthreshold autistic traits in the general population. Biological Psychiatry, 57(6), 655-660. https://doi.org/10.1016/j.biopsych.2004.12.014

Dapretto, M., Davies, M. S., Pfeifer, J. H., Scott, A. A., Sigman, M., Bookheimer, S. Y., \& lacoboni, M. (2006). Understanding emotions in others: mirror neuron dysfunction in children with autism spectrum disorders. Nature Neuroscience, 9(1), 28-30. https://doi.org/10.1038/nn1611

Dumas, G., Soussignan, R., Hugueville, L., Martinerie, J., \& Nadel, J. (2014). Revisiting mu suppression in autism spectrum disorder. Brain Research, 1585, 108-119. https://doi.org/10.1016/j.brainres.2014.08.035

Enticott, P. G., Kennedy, H. A., Rinehart, N. J., Bradshaw, J. L., Tonge, B. J., Daskalakis, Z. J., \& Fitzgerald, P. B. (2013). Interpersonal motor resonance in autism spectrum disorder: evidence against a global "mirror system" deficit. Frontiers in Human Neuroscience, 7, 1-8. https://doi.org/10.3389/fnhum.2013.00218

Enticott, P. G., Kennedy, H. A., Rinehart, N. J., Tonge, B. J., Bradshaw, J. L., Taffe, J. R., ... Fitzgerald, P. B. (2012). Mirror neuron activity associated with social impairments but not age in autism spectrum disorder. Biological Psychiatry, 71(5), 427-433. https://doi.org/10.1016/j.biopsych.2011.09.001

Fadiga, L, Fogassi, L., Pavesi, G., \& Rizzolatti, G. (1995). Motor Facilitation During Action Observation: A Magnetic Stimulation Study. Journal of Neurophysiology, 73(6), 26082611. Retrieved from http://jn.physiology.org/content/jn/73/6/2608.full.pdf

Fadiga, Luciano, Craighero, L., \& Olivier, E. (2005). Human motor cortex excitability during 
the perception of others' action. Current Opinion in Neurobiology, 15(2), 213-218. https://doi.org/10.1016/j.conb.2005.03.013

Fan, Y. T., Decety, J., Yang, C. Y., Liu, J. L., \& Cheng, Y. (2010). Unbroken mirror neurons in autism spectrum disorders. Journal of Child Psychology and Psychiatry and Allied Disciplines, 51(9), 981-988. https://doi.org/10.1111/j.1469-7610.2010.02269.x

Fishman, I., Datko, M., Cabrera, Y., Carper, R. A., \& Müller, R. A. (2015). Reduced integration and differentiation of the imitation network in autism: A combined functional connectivity magnetic resonance imaging and diffusion-weighted imaging study. Annals of Neurology, 78(6), 958-969. https://doi.org/10.1002/ana.24533

Fishman, I., Keown, C. L., Lincoln, A. J., Pineda, J. A., \& Müller, R.-A. A. (2014). Atypical cross talk between mentalizing and mirror neuron networks in autism spectrum disorder. JAMA Psychiatry, 71(7), 751-760. https://doi.org/10.1001/jamapsychiatry.2014.83

Forbes, P. A. G., Wang, Y., \& Hamilton, A. F. D. C. (2017). STORMy Interactions: Gaze and the Modulation of Mimicry in Adults on the Autism Spectrum. Psychonomic Bulletin and Review, 24(2), 529-535. https://doi.org/10.3758/s13423-016-1136-0

Gentner, R., \& Classen, J. (2006). Modular Organization of Finger Movements by the Human Central Nervous System. Neuron, 52(4), 731-742. https://doi.org/10.1016/J.NEURON.2006.09.038

Grèzes, J., Wicker, B., Berthoz, S., \& de Gelder, B. (2009). A failure to grasp the affective meaning of actions in autism spectrum disorder subjects. Neuropsychologia, 47(8-9), 1816-1825. https://doi.org/10.1016/j.neuropsychologia.2009.02.021

Hadjikhani, N., Joseph, R. M., Snyder, J., \& Tager-Flusberg, H. (2006). Anatomical Differences in the Mirror Neuron System and Social Cognition Network in Autism. Cerebral Cortex, 16(9), 1276-1282. https://doi.org/10.1093/cercor/bhj069

Hamilton, A. F. de C. (2013). Reflecting on the mirror neuron system in autism: A systematic review of current theories. Developmental Cognitive Neuroscience, 3, 91-105. https://doi.org/10.1016/j.den.2012.09.008

Hudac, C. M., Kresse, A., Aaronson, B., Deschamps, T. D., Webb, S. J., \& Bernier, R. A. (2015). Modulation of mu attenuation to social stimuli in children and adults with 16p11.2 deletions and duplications. Journal of Neurodevelopmental Disorders, $7(1)$. https://doi.org/10.1186/s11689-015-9118-5

Hudac, C. M., Stessman, H. A. F., DesChamps, T. D., Kresse, A., Faja, S., Neuhaus, E., ... Bernier, R. A. (2017). Exploring the heterogeneity of neural social indices for genetically distinct etiologies of autism. Journal of Neurodevelopmental Disorders, 9(1). https://doi.org/10.1186/s11689-017-9199-4

lacoboni, M. (2009). Imitation, empathy, and mirror neurons. Annual Review of Psychology, 60, 653-670. https://doi.org/10.1146/annurev.psych.60.110707.163604

lacoboni, M., \& Dapretto, M. (2006). The mirror neuron system and the consequences of its dysfunction. Nature Reviews Neuroscience, 7(12), 942-951. https://doi.org/10.1038/nrn2024

Kana, R. K., Wadsworth, H. M., \& Travers, B. G. (2011). A systems level analysis of the mirror neuron hypothesis and imitation impairments in autism spectrum disorders. Neuroscience and Biobehavioral Reviews, 35(3), 894-902. https://doi.org/10.1016/j.neubiorev.2010.10.007

Krings, T., Naujokat, C., \& Graf v. Keyserlingk, D. (1998). Representation of cortical motor function as revealed by stereotactic transcranial magnetic stimulation. 
Electroencephalography and Clinical Neurophysiology/Electromyography and Motor Control, 109(2), 85-93. https://doi.org/10.1016/S0924-980X(97)00078-7

Marsh, L. E., \& Hamilton, A. F. de C. (2011). Dissociation of mirroring and mentalising systems in autism. Neurolmage, 56(3), 1511-1519. https://doi.org/10.1016/j.neuroimage.2011.02.003

Martineau, J., Andersson, F., Barthélémy, C., Cottier, J. P., \& Destrieux, C. (2010). Atypical activation of the mirror neuron system during perception of hand motion in autism. Brain Research, 1320, 168-175. https://doi.org/10.1016/j.brainres.2010.01.035

Nishitani, N., Avikainen, S., \& Hari, R. (2004). Abnormal Imitation-Related Cortical Activation Sequences in Asperger's Syndrome. Annals of Neurology, 55(4), 558-562. https://doi.org/10.1002/ana.20031

Oberman, L. M., Enticott, P. G., Casanova, M. F., Rotenberg, A., Pascual-Leone, A., \& McCracken, J. T. (2016). Transcranial magnetic stimulation in autism spectrum disorder: Challenges, promise, and roadmap for future research. Autism Research, 9(2), 184203. https://doi.org/10.1002/aur.1567

Oberman, L. M., Hubbard, E. M., McCleery, J. P., Altschuler, E. L., Ramachandran, V. S., \& Pineda, J. A. (2005). EEG evidence for mirror neuron dysfunction in autism spectrum disorders. Cognitive Brain Research, 24(2), 190-198. https://doi.org/10.1016/j.cogbrainres.2005.01.014

Oberman, L. M., \& Ramachandran, V. S. (2007). The simulating social mind: The role of the mirror neuron system and simulation in the social and communicative deficits of autism spectrum disorders. Psychological Bulletin, 133(2), 310-327. https://doi.org/10.1037/0033-2909.133.2.310

Oldfield, R. C. (1971). The assessment and analysis of handedness: The Edinburgh inventory. Neuropsychologia, 9(1), 97-113. https://doi.org/10.1016/00283932(71)90067-4

Pokorny, J. J., Hatt, N. V., Colombi, C., Vivanti, G., Rogers, S. J., \& Rivera, S. M. (2015). The Action Observation System when Observing Hand Actions in Autism and Typical Development. Autism Research, 8(3), 284-296. https://doi.org/10.1002/aur.1445

Prinsen, J., \& Alaerts, K. (2019). Eye contact enhances interpersonal motor resonance: comparing video stimuli to a live two-person action context. Social Cognitive and Affective Neuroscience, 14(9), 967-976. https://doi.org/10.1093/scan/nsz064

Prinsen, J., \& Alaerts, K. (2020). Enhanced mirroring upon mutual gaze: multimodal evidence from TMS-assessed corticospinal excitability and the EEG mu rhythm. Scientific Reports, 10(1), 20449. https://doi.org/10.1038/s41598-020-77508-x

Prinsen, J., Bernaerts, S., Wang, Y., de Beukelaar, T. T., Cuypers, K., Swinnen, S. P., \& Alaerts, K. (2017). Direct eye contact enhances mirroring of others' movements: A transcranial magnetic stimulation study. Neuropsychologia, 95, 111-118. https://doi.org/10.1016/j.neuropsychologia.2016.12.011

Prinsen, J., Brams, S., \& Alaerts, K. (2018). To mirror or not to mirror upon mutual gaze, oxytocin can pave the way: A cross-over randomized placebo-controlled trial. Psychoneuroendocrinology, 90, 148-156. https://doi.org/10.1016/j.psyneuen.2018.02.016

Raymaekers, R., Wiersema, J. R., \& Roeyers, H. (2009). EEG study of the mirror neuron system in children with high functioning autism. Brain Research, 1304, 113-121. https://doi.org/10.1016/j.brainres.2009.09.068 
Rizzolatti, G., \& Sinigaglia, C. (2010). The functional role of the parieto-frontal mirror circuit: Interpretations and misinterpretations. Nature Reviews Neuroscience, 11(4), 264-274. https://doi.org/10.1038/nrn2805

Rossi, S., Hallett, M., Rossini, P. M., \& Pascual-Leone, A. (2012). Safety, ethical considerations, and application guidelines for the use of transcranial magnetic stimulation in clinical practice and research. Clinical Neurophysiology, 120(12), 323330. https://doi.org/10.1016/j.clinph.2009.08.016.Rossi

Ruysschaert, L., Warreyn, P., Wiersema, J. R., Oostra, A., \& Roeyers, H. (2014). Exploring the Role of Neural Mirroring in Children with Autism Spectrum Disorder. Autism Research, 7(2), 197-206. https://doi.org/10.1002/aur.1339

Schulte-Rüther, M., Otte, E., Adigüzel, K., Firk, C., Herpertz-Dahlmann, B., Koch, I., \& Konrad, K. (2017). Intact mirror mechanisms for automatic facial emotions in children and adolescents with autism spectrum disorder. Autism Research, 10(2), 298-310. https://doi.org/10.1002/aur.1654

Senju, A., \& Johnson, M. H. (2009). Atypical eye contact in autism: Models, mechanisms and development. Neuroscience and Biobehavioral Reviews, 33(8), 1204-1214. https://doi.org/10.1016/j.neubiorev.2009.06.001

Tanaka, J. W., \& Sung, A. (2016). The "Eye Avoidance" Hypothesis of Autism Face Processing. Journal of Autism and Developmental Disorders, 46(5), 1538-1552. https://doi.org/10.1007/s10803-013-1976-7

Théoret, H., Halligan, E., Kobayashi, M., Fregni, F., Tager-Flusberg, H., \& Pascual-Leone, A. (2005). Impaired motor facilitation during action observation in individuals with autism spectrum disorder. Current Biology, 15(3), 84-85. https://doi.org/10.1016/j.cub.2005.01.022

Wadsworth, H. M., Maximo, J. O., Donnelly, R. J., \& Kana, R. K. (2018). Action simulation and mirroring in children with autism spectrum disorders. Behavioural Brain Research, 341, 1-8. https://doi.org/10.1016/j.bbr.2017.12.012

Wang, Y., \& Hamilton, A. F. de C. (2012). Social top-down response modulation (STORM): a model of the control of mimicry in social interaction. Frontiers in Human Neuroscience, 6, 1-10. https://doi.org/10.3389/fnhum.2012.00153

Wang, Y., Newport, R., \& Hamilton, A. F. de C. (2011). Eye contact enhances mimicry of intransitive hand movements. Biology Letters, 7(1), 7-10. https://doi.org/10.1098/rsbl.2010.0279

Wang, Y., Ramsey, R., \& Hamilton, A. F. de C. (2011). The control of mimicry by eye contact is mediated by medial prefrontal cortex. Journal of Neuroscience, 31(33), 12001-12010. https://doi.org/10.1523/JNEUROSCI.0845-11.2011

Wechsler, D. (2012). WAIS-IV-NL: Wechsler Adult Intelligence Scale-Fourth EditionNederlandse Bewerking. Afname- en scoringshandleiding. Amsterdam: Pearson Assessment and Information.

World Medical Association. (2013). Declaration of Helsinki. Ethical Principles for Medical Research Involving Human Subjects. Journal of the American Medical Association, 310(20), 2191-2194. https://doi.org/10.1001/jama.2013.281053 